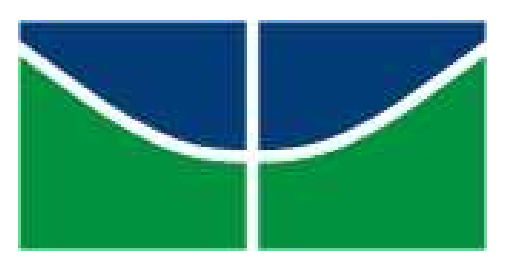

UNIVERSIDADE DE BRASÍLIA - UnB FACULDADE DE ECONOMIA, ADMINISTRAÇÃO, CONTABILIDADE E CIÊNCIA DA INFORMAÇÃO E DOCUMENTAÇÃO - FACE

\title{
AVALIAÇÃO DO NÍVEL DE ATIVIDADE FÍSICA DE SERVIDORES DA UNIVERSIDADE DE BRASÍLIA
}

HARLEY AZEVEDO JUNIOR

Brasília-DF

2010 


\title{
AVALIAÇÃO DO NÍVEL DE ATIVIDADE FÍSICA DE SERVIDORES DA UNIVERSIDADE DE BRASÍLIA
}

\begin{abstract}
Monografia apresentada à Faculdade de Economia, Administração, Contabilidade e Ciência da Informação e Documentação - FACE da Universidade de Brasília
\end{abstract}

Orientadora: KEILA ELIZABETH FONTANA

Brasilia-DF

2010 


\section{SUMÁRIO}

1- Introdução

Pág. 05

1.1 Justificativa

Pág. 07

1.2 Objetivos

Pág. 08

1.2.1 Geral

Pág. 08

1.2.2 Específico

Pág. 08

2- Metodologia

Pág. 09

2.1 - Coleta de dados

Pág. 09

3- Resultados

Pág. 12

4- Discussão

Pág. 16

5- Conclusão

Pág. 19

6- Referências Bibliográficas

Pág. 20

7- Anexos

Pág. 24 


\section{AGRADECIMENTOS}

Gostaria de prestar meus agradecimentos a todos aqueles que direta ou indiretamente participaram deste trabalho.

Em particular à Professora Keila Fontana, por sua orientação nesta monografia, sem a qual este trabalho não se realizaria.

Agradeço ao Professor Alexandre Rezende, por sua inestimável colaboração na interpretação do IPAQ.

Às pessoas que com boa vontade se propuseram a responder ao questionário, doando uma parte de seu tempo para participarem da pesquisa.

À minha esposa por me ajudar na revisão do trabalho. 


\section{INTRODUÇÃO}

Os índices de prevalência de sedentarismo nas Américas e em especial na América Latina não são tão claros, pois não temos levantamentos tão específicos e com metodologias similares e adequadas. Os levantamentos feitos por alguns países, geralmente em pesquisas ligadas a saúde pública, indicam dados que variam de país para país (MATSUDO et al., 2002).

Nos textos clássicos dos orientais, gregos e romanos já se mencionava a atividade física como um instrumento de manutenção e recuperação da saúde (MATSUDO et al. 2002).

Em relação aos estudos mais reconhecidos no Brasil sobre padrões de atividade física, ou prevalência de sedentarismo, o grupo de pesquisas do Centro de Estudos do Laboratório da Aptidão Física de São Caetano do Sul CELAFISCS, é o que mais tem apresentado dados em nível populacional. Em um estudo recentemente realizado por este grupo na população do estado de São Paulo, em uma amostra de 2001 indivíduos, verificou-se que 45,6\% das pessoas em São Paulo são insuficientemente ativas (ZAMAI et al. 2007). Nos Estados Unidos, de acordo com dados de CARSPERSEN et al. (1997), aproximadamente um em cada quatro adultos reportou ser fisicamente inativo (24\%). As mulheres reportaram ser mais inativas $(27 \%)$ do que os homens $(21 \%)$ e variações importantes ocorreram em função da etnia.

A prática de atividade física tem se mostrado benéfica na redução de fatores de risco, propiciando, por exemplo, melhora no metabolismo, controle de massa corporal e, muitas vezes o controle da hipertensão arterial. Essa prática contribui também para a manutenção de ossos, músculos e articulações saudáveis, estando ainda associada à prevenção de enfermidades como diabetes e osteoporose e alguns tipos de câncer (BARETTA et al., 2007).

A atividade fisica tem sido considerada um meio de preservar e melhorar o sedentarismo e estilos de vida que incorporam pouca atividade fisica.

Segundo Berlin, metanálise envolvendo mais de 40 estudos sugere que as doenças cardiovasculares são 1,9 vezes mais provavelmente 
desenvolvidas em sujeitos menos ativos que nos mais ativos fisicamente (BERLIN \& COLDITZ, 2005).

Entre portadores de fatores de risco predisponentes às disfunções crônico-degenerativas, a proporção de sujeitos classificados habitualmente como sedentários é significativamente maior que a de sujeitos ativos fisicamente (GUEDES et al., 2005).

Para a Organização Mundial de Saúde a prática de atividade física regular reduz o risco de morte prematura, doenças do coração. Atua na prevenção ou redução da pressão arterial, promove bem-estar, reduz o estresse, a ansiedade e a depressão (CEInfo, 2002-2003).

Podemos perceber que atualmente existe uma grande preocupação mundial em termos de qualidade de vida. A inatividade física (sedentarismo) é o fator de risco de doenças crônicas degenerativas não transmissiveis mais prevalentes na população, com conseqüências nocivas à saúde. Este levantamento tem sido alvo de muitos trabalhos ao redor do mundo, onde se tenta identificar o impacto causado pelo sedentarismo na vida do ser humano. Observa-se então que o sedentarismo não representa apenas um risco pessoal de enfermidades, mas um custo econômico para o indivíduo, sua família e toda a sociedade (ZAMAI et al., 2007).

Nas diretrizes clínicas estabelecidas pela Obesity Education Initiative Task Force (Força-Tarefa de Iniciativa de Educação Contra a Obesidade) do Instituto Nacional de Saúde (NIH) e do National Heart, Lung, and Blood Institute (Instituto Nacional do Coração, Pulmão e Sangue, 1998), sobrepeso e obesidade podem ser classificados a partir do índice de massa corporal. Indivíduos com IMC entre 25 a $29,9 \mathrm{~kg} / \mathrm{m}^{2}$ são classificados como indivíduos com sobrepeso; aqueles com IMC de $30 \mathrm{~kg} / \mathrm{m}^{2}$ ou mais são classificados como obesos (HEYWARD, 2004).

O Questionário Internacional de Atividade Física (IPAQ) foi proposto pelo grupo Internacional para Consenso de Medidas da Atividade Física, constituindo sob a chancela da Organização Mundial de Saúde (OMS), com representantes de 25 países, inclusive o Brasil (GUEDES et al., 2005) representado pelo CELAFISCS. 
Guedes et al., (2005) utilizaram o questionário de auto-administração em seu formato curto, por ser a versão mais frequentemente sugerida para utilização em populações jovens (esta versão possui 8 questões). Concluíram que ele pode ser aplicado em população maior de quatorze anos de idade.

Há que se considerar que para validação da versão 8 do IPAQ (MATSUDO, 2001), foram utilizados dados constantes do CELAFISCS desde 2000, no qual haviam maiores de 12 anos, de ambos os sexos, graus de escolaridade, níveis sócio-econômicos, profissões e cidades diversos. Foram obtidos resultados similares a outros instrumentos para medir o NAF, as formas curta e longa do questionário apresentaram reprodutibilidade similar, com os coeficientes de correlação variando de moderada a alta. Analisando a validade do questionário com os sensores de movimento, os coeficientes apresentaram valores de baixo a moderados tanto para versão curta quanto para a versão longa.

O IPAQ é um instrumento muito interessante e que têm promovido um grande esforço internacional para sua validação. Estes estudos de validação concluem por uma associação significativa com o registro de gasto energético (PARDINI et al., 2001). No entanto, são necessários mais estudos, principalmente em diferentes populações.

Já foram feitos alguns estudos com recém ingressos em universidades públicas (VIEIRA et al., 2002; SILVA et al., 2007), porém, há carência de estudos nesta área, e os que existem carecem de melhor aproveitamento e metodologia.

\subsection{JUSTIFICATIVAS}

Segundo Parsons et al., (1999) o envolvimento em atividades físicas regulares, é associado a uma menor incidência de obesidade, pressão e colesterol altos, entre outros.

Iniciativas em termos de saúde pública muitas vezes se deparam com a dificuldade de promover qualquer mudança de padrões comportamentais em adultos. A aquisição de hábitos saudáveis como alimentação adequada e 
prática de atividades físicas, se torna mais fácil à medida que são incentivados e consolidados na infância e adolescência.

Algumas dessas iniciativas têm sido realizadas com ênfase, devido aos comportamentos que o formam serem considerados aspectos fundamentais à promoção e manutenção da saúde, sendo a saúde entendida não apenas como a ausência de doença, mas como uma situação de bem-estar físico, mental e social (OMS, 1987).

Os comportamentos relacionados aos hábitos alimentares, nível de estresse, nivel de atividade física, relacionamentos, e comportamento preventivo são definidos como fatores que influenciam no bem-estar de indivíduos e grupos populacionais a curto e longo prazos (NAHAS, 2003).

Houve o interesse de pesquisar se os cursos oferecidos por esta instituição para a prática de atividades físicas são relevantes como fontes de beneficios para a saúde e a experiência com tais atividades, em especial aquelas sistematizadas, como importante fator influenciador do estilo de vida ativo do adulto. Esta monografia foi apresentada à Faculdade de

Economia, Administração, Contabilidade e Ciência da Informação e Documentação - FACE da Universidade de Brasília, do curso de Gestão Universitária, para a execução deste projeto.

\subsection{OBJETIVOS}

\subsubsection{Geral:}

Analisar o nível de atividade física (NAF) e o índice de massa corporal (IMC), dos servidores da Universidade de Brasília.

\subsubsection{Especificos:}

Classificar os servidores em categorias específicas quanto ao nível de prática de atividade fisica.

Verificar o conhecimento dos servidores a respeito da recomendação mínima de atividade física preconizada pelo Center for Disease Control/American College of Sports Medicine (CDC/ACSM). 


\section{METODOLOGIA}

Este estudo foi do tipo diagnóstico e a amostra foi constituída por 85 servidores do quadro da Universidade de Brasília, Professores e Técnicos Administrativos.

Segundo dados coletados no site da Universidade de Brasília (UnB), no ano de 2007 o quadro de servidores era constituído por 1297 professores e 2343 técnicos administrativos. Nossa expectativa de retorno dos formulários foi de $15 \%$ dos professores e de $20 \%$ dos técnicos administrativos.

Dividimos os grupos por idade, nas faixas de 19 a 35, 36 a 45 e de 46 a 69 anos de idade.

\subsection{Coleta de dados:}

A coleta de dados foi realizada através da distribuição de folhetos explicativos, no período de outubro a novembro de 2009, onde os servidores foram convidados a responder vinte e três perguntas do Questionário Internacional de Atividade Física - Versão 8 reduzida (IPAQ) de novembro de 2005. Como este trabalho foi realizado com seres humanos, se fez necessário a concordância do voluntário conforme o Termo de Consentimento e Livre Esclarecimento (TCLE), que foi enviado juntamente com o IPAQ. O retorno do TCLE não foi exigido, tendo em vista que o servidor ao responder o questionário estaria expressando sua concordância em participar.

Esta pesquisa foi aprovada quanto a sua ética científica pelo Comitê de Ética em Pesquisa Envolvendo Seres Humanos, da Faculdade de Ciências da Saúde da Universidade de Brasília (parecer N.102/2009), de acordo com as normas da Resolução 196/96 do Conselho Nacional da Saúde do Ministério da Saúde. A este Comitê cabe a solução ou encaminhamento de quaisquer questões éticas que possam surgir nessa pesquisa, de interesse do voluntário ou dos pesquisadores envolvidos.

Ao IPAQ foram acrescidas quatro perguntas referentes à estatura e a massa corporal e sobre o conhecimento da recomendação mínima de 
atividade física para a manutenção da saúde. Os questionários devidamente respondidos foram enviados para o e-mail do pesquisador responsável.

O Índice de Massa Corporal (IMC) foi calculado pelo pesquisador levando em consideração a estatura e massa corporal informadas pelo próprio servidor. O IMC foi calculado pela razão entre massa corporal e o quadrado da estatura e expressa em $\mathrm{kg} / \mathrm{m}^{2}$.

O IMC foi usado como uma ferramenta para identificar possiveis problemas de peso para adulto. Entretanto, o IMC não é uma ferramenta de diagnóstico. Por exemplo, a pessoa pode ter um IMC alto, porém para determinar se o excesso de peso é um risco para a saúde precisamos fazer mais análises que podem incluir medições de dobras cutâneas, atividade fisica, etc.

Para classificar o IMC foi utilizada o Quadro 1 abaixo apresentada pelo NHANES II survey (National Health and Nutrition Examination Survey) Pesquisa feita nos Estados Unidos entre 1976 e 1980.

Quadro 1 - Classificação do IMC para homens e mulheres.

\begin{tabular}{lcc}
\hline \multicolumn{1}{c}{ Condição } & $\begin{array}{c}\text { IMC em } \\
\text { Mulheres }\end{array}$ & $\begin{array}{c}\text { IMC em } \\
\text { Homens }\end{array}$ \\
\hline Abaixo do peso ideal & $<19,1$ & $<20,7$ \\
No peso ideal & $19,1-25,8$ & $20,7-26,4$ \\
Marginalmente acima do peso & $25,8-27,3$ & $26,4-27,8$ \\
Acima do peso ideal & $27,3-32,3$ & $27,8-31,1$ \\
Obeso & $>32,3$ & $>31,1$ \\
\hline
\end{tabular}

O nivel de atividade física (NAF) foi calculado pelo pesquisador utilizando o International Physical Activity Questionaire (IPAQ), na sua versão curta. Esta versão é composta de questões abertas e suas informações permitem estimar o tempo despendido por semana em diferentes dimensões de atividade física (caminhada e esforços físicos de intensidades moderada e vigorosa) e de inatividade física.

Atividades aeróbias de intensidade moderada, que são equivalentes a uma caminhada rápida e que pode acelerar o coração, poderá ser acumulada por no mínimo 30 min. e aceleradas nos últimos 10 minutos ou mais. Para 
Atividades vigorosas (intensas) podemos dar como exemplo o jogging (trote), o que causa um aumento rápido da ventilação pulmonar (respiração) e substancial aumento da freqüência cardíaca. Essa quantidade de exercício de atividade aeróbia é um adicional das recomendações das atividades diárias de intensidade leve (como, cuidados com a higiene, cozinhar, caminhada casual como ir ao shopping) ou para atividades que duram menos de 10 min. (ex: caminhada em casa, no escritório, ou voltando do estacionamento) (HASKELL et al., 2007).

O termo "dose" tem sido utilizado freqüentemente nas descrições em atividade fisica, mas pode ser interpretado de várias maneiras - como quantidade total de exercícios (energia total gasta) ou como intensidade, duração e freqüencia da atividade. Apesar de muitas pesquisas terem sido feitas sobre a quantidade total de atividade fisica (a qual pode ser utilizada para caracterizar os praticantes como "ativos", "moderadamente ativos", ou "sedentários-inativos, por exemplo), relativamente poucas pesquisas observacionais incluíram detalhes sobre o tipo de atividade praticada ou sua duração e freqüência de cada sessão. O total de atividade física é em função da intensidade, duração e freqüencia. Atividades físicas vigorosas (aquelas utilizando 9 a 6 METs) praticadas em duração e freqüência específicas geram gastos melhores de energia que atividades moderadas ( 3 a 6 METs) de mesma freqüência e duração (HASKELL et al., 2007).

Para tanto, foi realizada o produto entre a duração (minutos/dia) e a freqüência (dia/semana).

Para a análise do IPAQ, foi criado um programa na linguagem JAVA, tornando possivel a conversão do produto duração e freqüência informados em classificações dos níveis Leve, Moderado e Alto. Os dados coletados foram processados estatisticamente nos programas Microsoft Excel 2003 e SPSS 17.0, e utilizou-se a estatística descritiva para auxiliar a descrição e apresentação dos dados, tendo como base os cálculos de freqüência das respostas apresentadas nos questionários e seus respectivos percentuais. 


\section{RESULTADOS}

Dos 400 folhetos distribuidos aos técnicos administrativos, apenas 66 $(17,3 \%)$ responderam ao questionário. Ao passo que, dos 400 folhetos distribuídos aos professores apenas 16 (4\%) responderam. A amostra total de respondentes não correspondeu à espectativa inicial, e o baixo número de respostas possivelmente tenha ocorrido em função da greve dos servidores, ou opção por não responder por vergonha por estar acima do peso ideal ou até mesmo por falta de interesse em participar da pesquisa. Desta forma o tamanho da amostra ficou pequeno, e assim, optamos por fazer o agrupamento dos professores e técnicos, o que impossibilitou a realização da análise comparativa entre as categorias, como pretendido.

Além disso, o pequeno número de respondentes por categoria nos leva a acreditar na não representatividade da amostra e ainda a tendência dos dados, tendo em vista que tanto o IMC quanto no NAF, os individuos pesquisados apresentaram resultados acima dos esperados.

$\mathrm{Na}$ Tabela 1 foram avaliadas 43 mulheres que apresentaram os resultados de IMC relacionados por grupos etários. Constatamos que não foi encontrada nenhuma mulher abaixo do peso ideal. Quanto ao IMC, no peso ideal, as mulheres tiveram uma maior incidência na faixa etária mais baixa, tendo sido essa incidência de 32,5\%. Já em relação a marginalmente acima do peso ideal, houve uma igualdade entre as faixas etárias de 19 a 35 anos e a de 36 a 45 anos, que apresentaram o mesmo índice de 4,6\%. No nível acima do peso ideal, obtiveram uma concentração maior nas faixas de 36 a 45 anos e de 46 a 69 anos, ambas com 7,0\%. No nivel obeso, o índice foi de $4,6 \%$ somente na faixa de 19 a 35 anos.

A Tabela 2 apresenta os resultados de IMC do grupo masculino nas faixas etárias. Apenas 5,0\% dos mais novos (menor faixa etária) foram classificados como abaixo do peso ideal tendo apenas um homem $(2,0 \%)$ marginalmente acima do peso ideal na faixa de 46 a 69, contudo, no peso ideal observamos a maior incidência com $15,0 \%$ dos casos. 
Tabela 1. Distribuição das servidoras (mulheres) quanto ao IMC e faixa etária.

\begin{tabular}{|c|c|c|c|c|c|c|c|c|}
\hline CLASSIFICAÇÃO & IMC & $\begin{array}{c}\text { ANOS } \\
19 \text { a } 35\end{array}$ & $\%$ & $\begin{array}{c}\text { ANOS } \\
36 \text { a } 45\end{array}$ & $\%$ & $\begin{array}{c}\text { ANOS } \\
46 \text { a } 69\end{array}$ & $\%$ & $\begin{array}{c}\text { TOTAL } \\
\%\end{array}$ \\
\hline Abaixo do peso ideal & $<19,1$ & 0 & 0 & 0 & 0 & 0 & 0 & 0 \\
\hline No peso ideal & 19,1 a 25,8 & 14 & 32,5 & 7 & 16,2 & 8 & 18,6 & 67,3 \\
\hline $\begin{array}{l}\text { Marginalmente acima } \\
\text { do peso ideal }\end{array}$ & 25,8 a 27,3 & 2 & 4,6 & 2 & 4,6 & 1 & 2,3 & 11,5 \\
\hline Acima do peso ideal & 27,3 a 32,3 & 1 & 2,3 & 3 & 7,0 & 3 & 7,0 & 16,3 \\
\hline Obesa & $>32,3$ & 2 & 4,6 & 0 & 0 & 0 & 0 & 4,6 \\
\hline TOTAIS & & 19 & 44,0 & 12 & 28,0 & 12 & 28,0 & 100,0 \\
\hline
\end{tabular}

Tabela 2. Distribuição dos servidores (homens) quanto ao IMC e faixa etária.

\begin{tabular}{lcccccccc}
\hline \multicolumn{1}{c}{ CLASSIFICAÇÃO } & IMC & ANOS & $\%$ & ANOS & $\%$ & ANOS & $\%$ & TOTAL \\
$\%$ & a 35 & & 36 a 45 & & 46 a 69 & 0 & 0 \\
\hline Abaixo do peso ideal & $<20,7$ & 2 & 5,0 & 0 & 0 & 0 & 5,0 \\
No peso ideal & 20,7 a 26,4 & 3 & 7,5 & 8 & 20,0 & 8 & 20,0 & 47,5 \\
Marginalmente acima & 26,4 a 27,8 & 0 & 0 & 0 & 0 & 1 & 2,0 & 2,0 \\
do peso ideal & & & & & & & & \\
Acima do peso ideal & 27,8 a 31,1 & 3 & 7,5 & 3 & 7,5 & 6 & 15,0 & 30,0 \\
Obeso & $>31,1$ & 1 & 2,0 & 3 & 7,5 & 2 & 5,0 & 14,5 \\
\hline \multicolumn{1}{c}{ TOTAIS } & & 9 & 22,0 & 14 & 35,0 & 17 & 42,0 & 100,0 \\
\hline
\end{tabular}

Valores brutos e relativos (\%) ao número de respondentes.

A Tabela 3 apresenta a distribuição das mulheres quanto ao NAF e o IMC.

Tabela 3. Classificação do NAF quanto ao IMC das servidoras (mulheres).

\begin{tabular}{|c|c|c|c|c|c|c|c|c|}
\hline $\mathrm{IMC} \backslash \mathrm{NAF}$ & Valores & Leve & $\%$ & Moderado & $\%$ & Alto & $\%$ & $\begin{array}{c}\text { TOTAL } \\
\%\end{array}$ \\
\hline Abaixo do peso ideal & $<19,1$ & 0 & 0 & 2 & 4,8 & 1 & 2,4 & 7,2 \\
\hline No peso ideal & 19,1 a 25,8 & 2 & 4,8 & 6 & 15,0 & 17 & 41,0 & 60,8 \\
\hline $\begin{array}{l}\text { Marginalmente acima do } \\
\text { peso ideal }\end{array}$ & 25,8 a 27,3 & 1 & 2,4 & 2 & 4,8 & 2 & 4,8 & 12,0 \\
\hline Acima do peso ideal & 27,3 a 32,3 & 1 & 0 & 2 & 4,8 & 4 & 10,0 & 14,8 \\
\hline Obesa & $>32,3$ & 0 & 0 & 0 & 0 & 2 & 4,8 & 4,8 \\
\hline TOTAIS & & 4 & 7,2 & 12 & 29,4 & 26 & 63,0 & 100,0 \\
\hline
\end{tabular}

Marginalmente acima do nivel ideal houve a mesma incidência entre as mulheres nos niveis moderado e alto do NAF (4,8\%). Acima do peso ideal a maior concentração se deu no nível alto do NAF, com 10\%. Apesar de estarem acima do peso ideal, mostraram-se ativas. No total, em relação a todos os niveis do IMC, 63\% das mulheres apresentaram-se no nivel alto do NAF. Apenas duas mulheres, apesar de serem obesas, apresentaram nivel alto de atividade física. 
A Tabela 4 apresenta a classificação do NAF dos homens quanto ao IMC. Os servidores acima do peso ideal apresentaram niveis de atividade fisica moderado e alto com 10,0\% em cada. Da mesma forma, os servidores classificados como obesos apresentaram 7,5\% dos casos nos niveis moderado e alto do NAF. Um dado interessante é que tanto os homens (Tabela 4) quanto as mulheres (Tabela 3) com peso ideal do IMC apresentaram nível alto do NAF, sendo $30,0 \%$ dos homens e $41,0 \%$ das mulheres.

Tabela 4. Classificação do NAF quanto ao IMC dos servidores (homens)

\begin{tabular}{|c|c|c|c|c|c|c|c|c|}
\hline $\mathrm{IMC} \backslash \mathrm{NAF}$ & Valores & Leve & $\%$ & Moderado & $\%$ & Alto & $\%$ & $\begin{array}{c}\text { TOTAL } \\
\%\end{array}$ \\
\hline Abaixo do peso ideal & $<20,7$ & 0 & 0 & 0 & 0 & 2 & 5,0 & 5,0 \\
\hline No peso ideal & 20,7 a 26,4 & 0 & 0 & 7 & 17,5 & 12 & 30,0 & 47,5 \\
\hline $\begin{array}{l}\text { Marginalmente acima do } \\
\text { peso ideal }\end{array}$ & 26,4 a 27,8 & 0 & 0 & 0 & 0 & 3 & 7,5 & 7,5 \\
\hline Acima do peso ideal & 27,8 a 31,1 & 2 & 5,0 & 4 & 10,0 & 4 & 10,0 & 25,0 \\
\hline Obeso & $>31,1$ & 0 & 0 & 3 & 7,5 & 3 & 7,5 & 18,0 \\
\hline TOTAIS & & 2 & 5,0 & 14 & 35,0 & 24 & 60,0 & 100,0 \\
\hline
\end{tabular}

Na Tabela 5 apresentamos os resultados da classificação do NAF por sexo.

Os homens se concentraram mais no nivel moderado do NAF em relação às mulheres, tendo sua incidência de 34,0\% nesta categoria. Já as mulheres obtiveram vantagem em relação aos homens com 63,0\% delas apresentando nível alto de atividade física.

Podemos notar que 92,5\% dos homens e 90,8\% das mulheres, maioria esmagadora, encontraram-se entre os niveis moderado e alto, mostrando serem os servidores ativos, contudo, ambos os sexos apresentaram prevalência no alto nível do NAF. Fato esse bastante preocupante e sugere forte tendência das respostas colhidas.

A amostra pesquisada foi balanceada quanto a quantidade de mulheres (42) e homens (40). Os homens apresentaram maior incidência no nível obeso em relação às mulheres em todos os grupos etários. No peso ideal as mulheres estão em melhores condições em relação aos homens. No grupo de idades de 19 a 23 anos, as mulheres tiveram 33,0\% contra $7,5 \%$ para os homens. 
Tabela 5. Distribuição do nível de atividade física (NAF) por sexo.

\begin{tabular}{lcccc}
\hline \multicolumn{1}{c}{ NAF /SEXO } & HOMENS & $\%$ & MULHERES & $\%$ \\
\hline Leve & 3 & 7,5 & 4 & 9,3 \\
Moderado & 13 & 34,0 & 11 & 28,0 \\
Alto & 24 & 58,5 & 27 & 62,8 \\
\hline \multicolumn{1}{c}{ TOTAIS } & 40 & 100,0 & 42 & 100,0 \\
\hline \multicolumn{4}{c}{ Valores brutos e relativos (\%) } & ao número de respondentes.
\end{tabular}

Sobre o conhecimento dos servidores sobre a recomendação mínima de atividade física para a produção de saúde e a prevenção de doenças foram analisadas as respostas, levando-se em consideração o total subdividido em seus principais componentes, ou seja, tipo, freqüência, duração da sessão e intensidade da atividade.

Do total de oitenta e duas repostas recebidas, 44 (52,0\%) servidores responderam a esta questão, os outros 38 (48\%) não responderam. Entre os que responderam, 98,0\% poucos souberam sobre a recomendação mínima de atividade física necessária para a promoção de saúde e a prevenção de doenças. Sobre a duração dos exercícios 66\% das respostas estavam adequadas. Quanto ao tipo de atividade fisica 36,0\% descreveram quais atividades eram exercidas e apenas $18 \%$ se preocuparam com a intensidade, que é um fator importante para gerar respostas cardiorespiratórias suficientes para causar os benefícios esperados (HASKELL et al; 2007). Já a maioria se preocupou com a freqüência semanal da atividade (95,5\%).

O nivel de atividade física apresentou uma tendência semelhante entre os sexos quanto ao nível de atividade física, com um discreto, porém, maior envolvimento do sexo feminino em atividade fisica regular. 


\section{DISCUSSÃO}

O índice de massa corporal (IMC) é a medida internacional mais usada para calcular se uma pessoa está no peso ideal ou não. Ele foi desenvolvido pelo polímata Lambert Quételet no fim do século XIX. Trata-se de um método fácil e rápido para a avaliação do nível de gordura da pessoa, ou seja, é um preditor internacional de obesidade adotado pela Organização Mundial de Saúde - OMS (WHO/OMS, 1995).

Há alguns problemas em usar o IMC para determinar se uma pessoa está acima do peso. Por exemplo, pessoas musculosas podem ter um índice de massa corporal alto e não serem gordas. O IMC também não é aplicável para crianças, nem para idosos, aos quais seria necessário a aplicação de uma classificação diferenciada. Por exemplo, um grupo de assessoramento à Organização Mundial de Saúde concluiu que pessoas de origem asiática poderiam ser consideradas acima do peso com um IMC de apenas 23, enquanto nos ocidentais este nível é considerado como peso ideal (ZAMAI et al., 2007).

Apesar dos servidores apresentarem o IMC na faixa de normalidade, é necessária uma maior conscientização a respeito da prática sistematizada ou regular de exercícios físicos, como uma prevenção necessária que trará beneficios à saúde dos praticantes. Levando em consideração que além de preventivos são também terapêuticos para diversas enfermidades.

Com o avanço da tecnologia, tende-se a desencorajar a prática da atividade física (subir escadas rolantes ou de elevador ao invés de uma escada normal, andar pequenas distâncias de automóvel etc.), reduzindo o gasto energético necessário para o dia-a-dia, estimulando mais o sedentarismo que o trabalho ativo. (HASKELL et al., 2007).

As doenças acometidas pela falta regular de atividade fisica incluem doenças cardiovasculares, infarto por embolia, pressão alta, diabete do tipo II, osteoporose, obesidade, câncer de colo de útero, câncer de mama, ansiedade e depressão (HASKELL et al., 2007).

O aumento do nivel de atividade física da população tem sido alvo de muitos pesquisadores ao redor do mundo como forma de identificar o 
impacto do sedentarismo o estilo de vida do ser humano. Dados apresentados recentemente por VUORI (2001) revelam como o quadro do sedentarismo (tendo como critério a porcentagem de indivíduos que realizam menos de 3 horas por semana de atividade fisica recreacional) atinge tanto os países desenvolvidos como em desenvolvimento, afetando cerca de $57 \%$ da população européia (MATSUDO et al., 2002).

Os dados da prevalência do sedentarismo nas Américas e em especial na América Latina são menos claros, pois não temos levantamentos tão específicos e com metodologias similares e/ou adequadas (MATSUDO et al., 2002).

Nos Estados Unidos, de acordo com dados de CASPERSEM et al., (1997), aproximadamente um em cada quatro adultos reportou ser fisicamente ativo (24\%). As mulheres reportaram ser mais inativas (27\%) do que os homens $(21 \%)$ e variações importantes ocorrem em função da etnia.

Considerando os critérios de classificação usados neste estudo e, de acordo com os resultados podemos concluir que no geral o nível de atividade física foi similar em ambos os sexos, com uma semelhança no nível leve do NAF, sendo que no nível moderado do NAF os homens tiveram uma diferença de $6,0 \%$ a mais que as mulheres e, no nível Alto do NAF as mulheres se sairam melhor com $4,3 \%$ a mais que os homens.

Nossos dados diferem dos apresentados por CASPERSEM et al., porque nosso grupo apresentou a maioria dos servidores ativos (92,5\% dos homens e 90,8\% das mulheres). Este fato demonstra uma evidente tendência, provavelmente devido ao nosso número de respondestes ser pequeno. Devemos cogitar ainda que apenas responderam ao questionário aqueles que se sentiram a vontade para afirmar que eram ativos e estavam próximos do seu peso ideal. Infelizmente, não poderemos diante desses dados, arriscar que os resultados sejam frutos da alta escolaridade dos respondentes. Estudos nesta área (COSTA, 2010) revelaram que as pessoas com mais anos de estudo são as que mais praticam atividades físicas de lazer. Contudo, no nosso caso, não foi medido o nivel de escolaridade dos servidores, principalmente dos técnicos administrativos. O nível de escolaridade desses pode variar desde o nível médio ao pós-graduado. 
Portanto, nos resta sugerir que mais estudos sejam realizados para esclarecer se realmente os servidores da Universidade de Brasília são ativos e privilegiados pela alta escolaridade, conseguindo assim se manterem nos níveis de peso ideal.

Em 1995, foram publicadas pelo Centro de Controle de Prevenção de Doenças Americano (CDC) e o ACSM recomendações para adultos americanos, os quais deviam acumular 30 minutos ou mais de atividade física de intensidade moderada na maioria ou em todos os dias da semana. O propósito destas recomendações foi encorajar o aumento da participação em atividades físicas da população americana sedentária (HASKELL et al., 2007).

Levando em consideração estes aspectos, a maioria os servidores se preocupou apenas com a duração dos exercícios, não levando tanto em consideração a intensidade e o tipo, o que vai de encontro com a recomendação do $\mathrm{CDC} / \mathrm{ACSM}$, ou seja, "que todo individuo deve acumular ao menos 30 minutos de atividade física, na maioria dos dias da semana, em intensidade moderada, de forma contínua ou acumulada”.

Com relação à reprodutibilidade das informações apresentadas pelos respondentes, sugere-se que a versão do IPAQ (MATSUDO et al., 2002) apresenta satisfatória capacidade de concordância entre réplicas de sua publicação, confirmando resultados apresentados por outros estudos disponibilizados na literatura no sentido de que a reprodução envolve esforços físicos moderados e vigorosos sendo maior que a das informações relacionadas às demais dimensões de atividade física.

Como a nossa amostra foi significativa, mas não representativa, se faz necessário um estudo mais abrangente a fim de podermos afirmar estas evidências encontradas no referido trabalho. 


\section{CONCLUSÃo}

Concluímos que ambos os sexos apresentaram no peso ideal do IMC uma maior predominância no nivel alto do NAF, com respectivamente $41,0 \%$ das mulheres e $30,0 \%$ dos homens.

Em ambos os sexos houve prevalência de indivíduos muito ativos.

O público pesquisado foi bastante homogêneo, homens e mulheres apresentaram-se ser ativos. Vale destacar também o baixo percentual de indivíduos classificados como leves no NAF.

Os servidores, em maioria, apresentaram-se ativos com 92,5\% dos homens e 90,8\% das mulheres. Este fato demonstra uma evidente tendência, provavelmente devido a nossa amostra ser pequena. Devemos supor ainda que apenas responderam ao questionário aqueles que se sentiram a vontade para afirmar que eram ativos e estavam próximos do seu peso ideal.

Em relação ao peso ideal do IMC as mulheres tiveram $32,5 \%$ de incidência nas idades de 19 a 35 anos, contra $20,0 \%$ dos homens na faixa de 36 a 69 anos.

No NAF em relação ao IMC no peso ideal as mulheres obtiveram uma maior incidência com $41,0 \%$ contra $30,0 \%$ dos homens.

No NAF em relação ao sexo com $62,8 \%$ as mulheres apresentaram-se no nivel alto e os homens com 58,5\%.

Concluímos que em relação a recomendação do CDC/ACSM, a maior parte dos servidores se preocupou com a duração dos exercícios, não levando tanto em consideração a intensidade e o tipo de exercícios.maioria se preocupou apenas com a duração dos exercícios, não levando tanto em consideração a intensidade e o tipo.

Com relação à reprodutibilidade das informações apresentadas pelos respondentes, sugere-se que a versão do IPAQ (MATSUDO et al., 2002) apresenta satisfatória capacidade de concordância entre réplicas de sua publicação. 
Como a nossa amostra foi significativa, mas não representativa, se faz necessário um estudo mais abrangente a fim de podermos afirmar estas evidências encontradas no referido trabalho.

Em razão desta pesquisa ser tendenciosa, não pudemos obter o conhecimento necessário sobre o nível do NAF dos servidores. Sendo assim não temos como sugerir medidas adequadas para melhorias ou não na condição físicas dos servidores. 


\section{REFERÊNCIAS BIBLIOGRÁFICAS}

BARRETA, E; BARRETA, M; PERES, KG. 2007. Nivel de atividade fisica e fatores associados entre adultos no Município de Joaçaba. Cadernos de Saúde. 23(7).

BERLIN, JA; COLDITZ, GA. 1990. A meta-analysis of physical activity in the prevention of coronary heart disease. Am J Epidemiol.132:612-28.

CARSPERSEN, CJ; ZACK, MM. 1997. The prevalence of physical inactivity in the United States. In: Physical Activity and Cardiovascular Health - A National Consensus Human Kinectics.

Centers for Disease Control and Prevention. USA, 1991. Prevalence of sedentary life-style: behavioral risk factor surveillance system. Morb Mort Wkly Rep. 1993; 42:576-9.

COSTA, EF. 2010. Mestrado intitulado Prática de atividade fisica e sua relação com escolaridade em adultos de Ermelino Matarazzo. USP. São Paulo.

DATA FOLHA. 1997. Pesquisa de prática de atividades físicas no Brasil. Relatório Data Folha, novembro.

GOMES, VB; SIQUEIRA, KS; SICHIERI, R. 2001. Atividade física em amostra probabilística da população do Município do Rio de Janeiro. Cadernos de Saúde Pública. 17(4): 969-979.

GUEDES, DP; LOPES, CC; GUEDES, JERP. 2005. Reprodutividade e validade do Questionário Internacional de Atividade Física em adolescentes. Revista Brasileira de Medicina Esportiva. 11(2). 
MATSUDO, SM; MATSUDO, VC; ARAÚJO, T; ANDRADE, D; ANDRADE, E; OLIVEIRA, L; BRAGGION, G. 2002. Nivel de atividade física da população do Estado de São Paulo: análise de acordo com o gênero, idade, nível socioeconômico, distribuição geográfica e de conhecimento. Revista Brasileira de Ciência e Movimento. 10(4); 41-50.

HAHAS, M. 2003. Atividade física, saúde e qualidade de vida: Conceitos e sugestões para um estilo de vida ativo. Londrina: Mediograf.

HASKELL, WL; LEE, I-MIN; PATES, RR; POWWLL, KE; BLAIR, SN; FRANKLIN, BA; MACERA, CA; HEATH, GW; THOMPSONS, PD; BAUMAN, A. 2007. Physical Activiti and Public Health: Updated Recommendation for Adults from the American College of Sports Medicine and the American Heart Association. 39(8):1423-1434.

HEYWARD, VH. 2004. Avaliação Física e Prescrição de Exercício - Técnicas Avançadas. Ed. Artmed, p. 25-26.

IPAQ - International Physical Activity Questionnaire. Guidelines for Data Procesing and Analysis of the International Physical Activity Questionnaire (IPAQ) - Short and Long Forms, November 2005.

OMS - Organização Mundial e Saúde. 1987. Carta de Otawa para La Promoción y Educación para La Salud. Rev Sanid Hig Publica. 61: 129-39.

PARSONS, TJ; POWER C; LOGANS S; SUMERBELL, CD. 1999. Childhood predictors of adult obesity: a systemic review. Int J Obesity. 23 (supply. 8), s1-s7.

PARDINI, R; MATSUDO, S; ARAÚJO, T et al. 2001.Validação do questionário internacional de nível de atividade física IPAQ - versão 6: estudo piloto em adultos jovens brasileiros. Revista Brasileira Ciência \& Movimento 9(3): 4551. 
Prefeitura do Município de São Paulo. Secretaria Municipal de Saúde. Coordenação e Epidemiologia e Informação, CEInfo. 2002-2003. Inquérito Domiciliar sobre Comportamentos de Risco e Morbidade Referida de Doenças e Agravos não Transmissiveis. Brasil, 15 capitais e Distrito Federal.

VIEIRA, VCR; PRIORE, SE; RIBEIRO, SMR. 2002. Perfil socioeconômico, nutricional e de saúde de adolescentes recém-ingressos em uma universidade pública brasileira. Revista de Nutrição.15(3):272-282.

WHO. 1995. Physical status: the use and interpretation of anthropometry. Report of a WHO Expert Committee. WHO Technical Report Series 854. Geneva: World Health Organization.

ZAMAI, CA; RODRIGUES, AA; BANKOFF, ADP; DELGADO, MA; BRAGA, LES; FILOCOMO, M. 2007. Análise do Nivel de Atividade Física de Estudantes do Curso de Educação Física da Universidade Paulista. Movimento \& Percepção 8(11). 
7. ANEXOS

7.1 Convite para participação no trabalho

7.2 IPAQ

7.3 TCLE 
Universidade de Brasília

Faculdade de Educação Física

O senhor (a) está sendo convidado (a) a participar da pesquisa "Índice de Massa Corporal e Nível de Atividade Física dos Servidores da Universidade de Brasília". Para participar, basta acessar o site www.harleyhaj.ueuo.com, e preencher o questionário em anexo. O senhor (a) não levará mais do que cinco minutos.

Atenciosamente,

Harley Azevedo Junior

harley@unb.br 


\section{QUESTIONÁRIO INTERNACIONAL DE ATIVIDADE FÍSICA -} VERSÃO CURTA

Nome:

Data: $\quad l \quad l \quad$ Sexo: F( ) $\mathrm{M}($ )

Professor ( ) Téc. Administrativo ( ) Data de Nascimento:

Nós estamos interessados em saber que tipos de atividade física as pessoas fazem como parte do seu dia a dia. Este projeto faz parte de um estudo para monografia do Curso de Especialização em Gestão Universitária. Suas respostas nos ajudarão a entender que tão ativos nós somos. As perguntas estão relacionadas ao tempo que você gasta fazendo atividade física na ÚLTIMA semana. As perguntas incluem as atividades que você faz no trabalho, para ir de um lugar a outro, por lazer, por esporte, por exercício ou como parte das suas atividades em casa ou no jardim. Suas respostas são MUITO importantes. Por favor responda cada questão mesmo que considere que não seja ativo. Obrigado pela sua participação!

Para responder as questões lembre que:

- atividades físicas VIGOROSAS são aquelas que precisam de um grande esforço físico e que fazem respirar

MUITO mais forte que o normal

atividades físicas LEVES ou MODERADAS são aquelas que precisam de algum esforço físico e que fazem

respirar UM POUCO mais forte que o normal

Para responder as perguntas pense somente nas atividades que você realiza por pelo menos 10 minutos contínuos de cada vez.

1. Em quantos dias de uma semana normal, você realiza atividades LEVES ou MODERADAS? Como nadar, pedalar ou varrer:
(a) dias por SEMANA
(b) Não quero responder
(c) Não sei responder

2. Nos dias em que você faz este tipo de atividade, quanto tempo você gasta fazendo essas atividades POR DIA?
(a) _ horas __ minutos
(b) Não quero responder
(c) Não sei responder

3. Em quantos dias de uma semana normal, você realiza atividades VIGOROSAS? Como correr e nadar rápido ou fazer jogging:
(a) __ dias por SEMANA
(b) Não quero responder
(c) Não sei responder

4. Nos dias que você faz este tipo de atividades quanto tempo você gasta fazendo essas atividades POR DIA?
(a) horas minutos
(b) Não quero responder
(c) Não sei responder

ATIVIDADE FÍSICA NO TRABALHO

5. Atualmente você trabalha ou faz trabalho voluntário fora de sua casa?

Sim ( ) Não ( )

6. Quantos dias de uma semana normal você trabalha? dias

7. Durante um dia normal de trabalho, quanto tempo você gasta:

Andando rápido: horas minutos

8. Fazendo atividades de esforço MODERADO como subir escadas ou carregar pesos leves: horas minutos

9. Fazendo atividades VIGOROSAS como trabalho de construção pesada ou trabalhar com enxada, escavar: horas minutos

\section{ATIVIDADE FÍSICA EM CASA}

Agora pensando em todas as atividades que você tem feito em casa durante uma semana normal:

10. Em quantos dias de uma semana normal você faz atividades dentro da sua casa por pelo menos 10 minutos de esforço MODERADO como aspirar, varrer ou esfregar:

(a) dias por SEMANA

(b) Não quero responder

(c) Não sei responder

11. Nos dias que você faz este tipo de atividade quanto tempo você gasta fazendo essas atividades POR DIA? horas minutos

12. Em quantos dias de uma semana normal você faz atividades no jardim ou quintal por pelo menos 10 minutos de esforço MODERADO como varrer, rastelar, podar:

(a) __ dias por SEMANA

(b) Não quero responder

(c) Não sei responder 
13. Nos dias que você faz este tipo de atividades quanto tempo você gasta POR DIA? minutos

14. Em quantos dias de uma semana normal você faz atividades no jardim ou quintal por pelo menos 10 minutos de esforço VIGOROSO como capinar arar, lavar o quintal:
(a) __ dias por SEMANA
(b) Não quero responder
(c) Não sei responder

15. Nos dias que você faz este tipo de atividades quanto tempo você gasta POR DIA? minutos

\section{ATIVIDADE FÍSICA COMO MEIO DE TRANSPORTE}

Agora pense em relação a caminhar ou pedalar para ir de um lugar a outro em uma semana normal.

16. Em quantos dias de uma semana normal você caminha de forma rápida por pelo menos 10 minutos para ir de um lugar para outro? (Não inclua as caminhadas por prazer ou exercício)
(a) dias por SEMANA
(b) Não quero responder
(c) Não sei responder

17. Nos dias que você caminha para ir de um lugar para outro quanto tempo POR DIA você gasta caminhando? (Não inclua as caminhadas por prazer ou exercício) horas minutos

18. Em quantos dias de uma semana normal você pedala rápido por pelo menos 10 minutos para ir de um lugar para outro? (Não inclua o pedalar por prazer ou exercício)
(a) __ dias por SEMANA
(b) Não quero responder
(c) Não sei responder

19. Nos dias que você pedala para ir de um lugar para outro quanto tempo POR DIA você gasta pedalando? (Não inclua o pedalar por prazer ou exercício) horas minutos
20. Peso corporal $(\mathrm{kg})$

Estatura $(\mathrm{cm})$

21. Você conhece a recomendação mínima de atividade física necessária para a promoção de saúde e a prevenção de doenças:

( ) $\operatorname{Sim}$ ( ) Não

22. Caso afirmativo, você poderia resumir qual é a recomendação mínima de atividade física semanal preconizada para uma boa saúde?

23. Seu e_mail 
Universidade de Brasília/Faculdade de Economia, Administração, Contabilidade e Ciências da Informação - FACE/UnB

Curso: Especialização em Gestão Universitária

Pesquisa: Índice de Massa Corporal e Nível de Atividade Física dos Servidores da Universidade de Brasília

\section{TERMO DE CONSENTIMENTO E LIVRE ESCLARECIMENTO}

O Senhor (a) está sendo convidado a participar da pesquisa "Índice de massa corporal e nivel de atividade física dos servidores da Universidade de Brasília”, o senhor (a) deverá, após ter lido, e compreendido totalmente o presente termo de consentimento, responder o questionário em anexo.

O questionário consta de 23 questões que poderá levar no máximo 5 minutos para o seu preenchimento. Seu nome e identificação serão resguardados e, não serão divulgados, o resultado final do trabalho the será enviado por e-mail.

Não será necessária a sua assinatura para aceitar este termo. O envio do questionário devidamente preenchido terá validade como tal.

$\mathrm{O}$ estudo tem como objetivo avaliar o indice de massa corporal (IMC) e o nível de atividade física dos servidores da UnB. Busca contribuir na descrição do perfil da massa corporal e do nível de atividade física do servidor, possibilitando auxiliar na elaboração de politicas de incentivo para a prática de atividades físicas preventivas e auxiliar na validação do questionário de atividade fisica utilizado.

Nenhum tipo de pagamento será efetuado pela sua participação como voluntário.

Todos os procedimentos da pesquisa serão tabulados na Faculdade de Educação Física da Universidade de Brasília.

Esta pesquisa foi aprovada quanto a sua ética científica pelo Comitê de Ética em Pesquisa Envolvendo Seres Humanos, da Faculdade de Ciências da Saúde da Universidade de Brasília (parecer N.102/2009), de acordo com as normas da Resolução 196/96 do Conselho Nacional da Saúde do Ministério da Saúde. A este Comitê cabe a solução ou encaminhamento de quaisquer questões éticas que possam surgir nessa pesquisa, de interesse do Voluntário ou dos Pesquisadores envolvidos.

Brasilia, de de 2009

Pesquisador Responsável: Harley Azevedo Junior

E-mail: harley@unb.br

Orientando: Keila Elizabeth Fontana

Telefones para contato:

Pesquisador responsável: 3107.2521 / 8131.9580

Comitê de Ética da FS: 3307.3799 